\title{
Ovarian function in domestic ruminants: Mechanistic and translational aspects
}

\author{
B.K. Campbell ${ }^{1 *}$, J. Hernandez-Medrano ${ }^{1}$, A.S. McNeilly ${ }^{3}$, R. Webb ${ }^{4}$ \\ and H.M. Picton ${ }^{5}$
}

\begin{abstract}
${ }^{1}$ Division of Child Health Obstetrics and Gynaecology, School of Medicine, University of Nottingham, Nottingham, NG7 2UH, UK; ${ }^{2}$ Department of Veterinary Basic Sciences, The Royal Veterinary College, Hawkshead Lane, Herts AL9 7TA, United Kingdom; ${ }^{3}$ MRC Centre for Reproductive Health, University of Edinburgh, Edinburgh EH16 4TJ; ${ }^{4}$ Division of Animal Science, School of Biosciences, University of Nottingham, Loughborough Leicestershire, LE12 5RD, UK; ${ }^{5}$ Reproduction and Early Development,

Leeds Institute of Genetics, Health and Therapeutics, University of Leeds, Leeds, LS2 9JT, UK
\end{abstract}

\begin{abstract}
The purpose of this review is to illustrate the critical value of integration of both in vitro and whole animal approaches in order to make real progress in terms of understanding the underlying mechanisms controlling ovarian follicle development and also in utilising domestic ruminants as models for the human in the translational development of interventions to address infertility. In terms of mechanistic aspects, this review concentrates on interactions between members of the TGF $\beta$ super family derived from both somatic cells (AMH) and the oocyte (BMP6, BMP15, GDF9) and illustrates how integration of in vitro and in vivo approaches has allowed us to propose a functional relationship between these factors in regulating the progression of follicles through the gonadotrophin-responsive to dependent phases of follicle development. In this section, a new in situ direct ovarian perfusion system is introduced.. In terms of translational aspects, this review details the integration of in vitro and in vivo approaches in order to develop methods in sheep that allow high rates of restoration of ovarian function and fertility following whole ovarian cryopreservation and autotransplantation in adult animals. This methodology should be of value to women at risk of premature ovarian failure. It is concluded that, given the vastly increased cost of ruminant research in developed countries, that meetings such as the RRS can play a critical role in encouraging international collaboration in order to ensure the continued exploitation of monovulatory ruminants as valuable experimental models.
\end{abstract}

\section{Introduction}

It is now well established that ovarian follicle and oocyte development is controlled by a complex array of autocrine and paracrine factors secreted by both the oocyte and follicular somatic cells which interact to initially control follicle development locally during initial gonadotrophin-independent stages 
of follicles development and then increasingly act to modulate the responsiveness of ovarian granulosa and theca cells to the pituitary gonadotrophins through the subsequent gonadotrophin-responsive and -dependent stages of follicle development (Campbell et al., 1995; Webb and Campbell, 2007a; Webb et al., 1999). Advances in the development of physiological, serum-free culture systems for preantral follicles (Picton et al., 2003), somatic cells (Campbell et al., 1996; Campbell et al., 1998) and oocytes (Picton et al., 2003) allied with the enormous power of the "omic" technologies has resulted in the implication of a vast array of local factors from a range of major local regulatory systems (see below) in the control of major development checkpoints during follicle development. The complex nature of these inter-relationships between interacting systems has led to a degree of uncertainty as to the actual identity of the key regulatory systems controlling follicular development and this problem is compounded by contradictory evidence, much of which can be attributed to species differences in the role and structure of the same local factors/system.

This problem is particularly evident in ruminant reproduction research, where despite the economic value of these species and their usefulness as experimental models for the human, there is limited commercial availability of species specific reagents and/or sequence information when compared to polyovular rodents and the human. Further, the current lack of stem cell and specific knock-out technology severely limits our ability to conduct the type of interventionist in vivo studies required to elucidate key development pathways in ruminant species. In this regard it is telling that some of the major advances in ruminant reproduction research in the last decade have come from identification of the naturally occurring mutations in sheep which were known to result in infertility and/or increased prolificacy (McNatty et al., 2005).

Prof George Eric Lamming was an outstanding animal physiologist who understood the need to refer to the whole animal in order to make real progress in understanding reproductive processes that will in turn lead to advances to boost production and/or treat infertility. It is therefore appropriate that the main objective of this review is to emphasise the critical nature of integration of both in vitro and in vivo approaches in ruminant reproduction research, despite the vastly increased cost of this type of research (at least in the UK). Thus after a brief summary of folliculogenesis, we plan to illustrate this point through recent research from our laboratories which will emphasise the value of some classic technology and also introduce some new in vivo models to facilitate both mechanistic and translational aspects of ovarian research. It should be emphasised that this review is not intended to cover the whole of the complex endocrine and local regulatory system controlling follicle development but rather to highlight some aspects of these local regulatory systems in order to illustrate the value of an integrated experimental approach.

\section{Ovulatory follicle development}

Ovarian folliculogenesis is a lengthy and intricately regulated process marked by dramatic proliferation and precisely orchestrated differentiation of both the somatic and germ cell elements. The ovarian follicle is the fundamental developmental unit of the mammalian ovary, consisting of a germ cell (oocyte) in close association with somatic cells (granulosa cells). Primordial follicles represent the source from which follicles will be recruited for growth throughout life and as the paired ovaries of an individual contain around 100,000-250,000 of these follicles at birth (Cahill, 1981; Turnbull et al., 1977). Once follicles have been initiated to grow, the granulosa cells proliferate to form multilaminar structures (preantral follicles) which subsequently form a fluid filled space (antrum) and a well differentiated theca layer (Young and McNeilly, 2010). Follicular development in humans, sheep and cattle is a lengthy process, taking around 4-6 months (Turnbull et al., 1977; Webb and Campbell, 2007a) with the majority of this time (4 months) being spent in the preantral stages of development. Gap junction-mediated communication between the oocyte and the surrounding somatic cells 
is essential for the coordinated development of both cell types, making folliculogenesis a highly synchronised process. The factors that initiate this process, the recruitment of primordial follicles, remain unknown although a large number of potential modulators have been identified (Picton et al., 2008; Tingen et al., 2009). Following follicle recruitment, the oocyte enlarges, accumulating maternal transcripts, proteins and organelles that will support embryonic development. At the same time, the granulosa cells change shape and start to proliferate and differentiate, becoming responsive to gonadotrophic signals (Picton et al., 2008). Oocyte-somatic cell communication is maintained throughout follicle growth, during which time somatic cells provide the oocyte with metabolic substrates and meiosis-arresting signals (Picton et al., 2008). When the follicle reaches a diameter of $200-400 \mu \mathrm{m}$, the antrum develops and this is followed by widespread atresia (50-70\% for follicles over $1 \mathrm{~mm}$ ) so that the vast majority (>99\%) of follicles fail to ovulate. Follicle development from the late preantral stage to the small antral stage ( $2 \mathrm{~mm}$ in sheep, $4-5 \mathrm{~mm}$ in cattle and humans) is termed gonadotrophin-responsive because although development of these follicles can be influenced by FSH, the growth of follicles to these stages still occurs in the absence of FSH (Dufour et al., 1979; Webb and Campbell, 2007a). The transition from the gonadotrophin-responsive to the subsequent gonadotrophin-dependent phase is characterised by a dramatic decline in the mitotic index of the ovarian somatic cells (Fig. 1) as differentiation of these cells occurs. Further development of antral follicles, however, is termed gonadotrophin-dependent as FSH must be maintained above a critical threshold concentration (Baird, 1983; Webb et al., 1999) in order to support growth to an

A. Oocyte diameter

\section{B. Maximum period $\mathrm{AMH}$ expression}
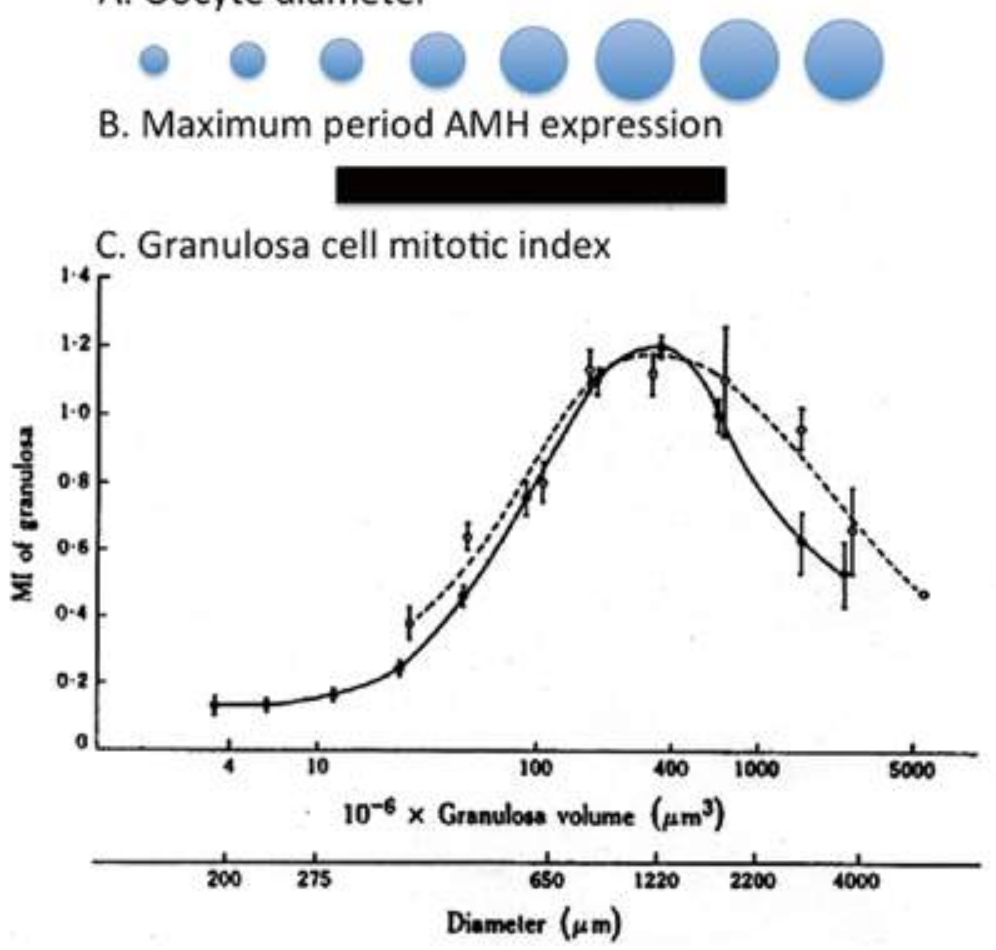

Fig. 1. Schematic diagram illustrating changes in oocyte diameter $(A)$ and relative level of maximal $\mathrm{AMH}$ expression (B) in relation to changes in granulosa cell mitotic index during follicle development in sheep (C). Note that majority of oocyte growth occurs prior to rapid increase in mitotic index in somatic cells which is temporarily related to pattern of $\mathrm{AMH}$ expression. C redrawn from Turnbull et al., 1977. 
ovulatory size, at which point the follicle is highly vascularised, the granulosa cells have high levels of aromatase and LH receptor expression and contains a cytoplasmically mature, developmentally competent oocyte. The mechanisms regulating the recruitment, selection and dominance of the ovulatory follicle in monovulatory species has been the subject of intensive investigation and the occurrence of spontaneous mutations in sheep which result in dysregulation of this process, such as the FecB mutation (see below) are valuable experimental models for elucidating the underlying mechanisms of this selection process.

The role of potential ligands for BMPR1B (Alk6) on ovine follicle development

In the following section we will relate some recent work from our laboratories in order to illustrate the value of integration of both in vitro cell culture and analytical techniques with whole animal studies to ensure significant progress in our understanding of the mechanisms regulating follicular development. The initial impetus for this work came from the identification of the FecB Booroola mutation, which resulted in marked increases in ovulation rate due to precocious ovulatory follicle maturation, as being due to a single point mutation in the intracellular domain of one of the receptors for bone morphogenetic proteins (BMPR1B, also known as Alk6) (Mulsant et al., 2001; Souza et al., 2001; Wilson et al., 2001). We have previously presented evidence to suggest that the FecB mutation exerts its action at the level of the ovary (Campbell et al., 2003a) rather than modulating pituitary gonadotrophin release (McNatty and Henderson, 1987), and more recent data in which depression in FSH levels was shown to negate the effect of the FecB mutation on prolificacy, confirmed this hypothesis by providing unequivocal evidence that the FecB mutation acts through modulation of gonadotrophic signals (AS McNeilly, DT Baird BK Campbell unpublished results). Our resultant studies on potential ligands for Alk6, including bone morphogenetic protein (BMP) 6, anti-mullerian hormone (AMH) and BMP15 (Miyazono et al., 2005; Shi and Massague, 2003), described below, have revealed a much wider range of actions for these factors than those associated with just ovulatory follicle selection. Readers are referred to a number of excellent reviews for detailed descriptions of the BMP system in the ovary (Juengel et al., 2004a; Knight and Glister, 2006; McNatty et al., 2005).

\section{BMP6}

Initial cell culture studies in sheep showed that human recombinant BMP2, BMP4 and BMP6 were equally potent enhancers of $\mathrm{FSH}$-induced oestradiol production by sheep granulosa cells whilst in theca cells (TC), similar doses of these BMPs $(5-50 \mathrm{ng} / \mathrm{ml})$ inhibited $\mathrm{LH}$-stimulated androstenedione (A4) production whereas lower doses $(0.005-0.05 \mathrm{ng} / \mathrm{ml})$ stimulated TC proliferation and hence total androstenedione production (Campbell et al., 2006). Similar findings to these had also been made in the bovine (Glister et al., 2005). In order to resolve these contradictory findings, BMP6, which had been identified as the most highly expressed BMP in sheep GC (Campbell et al., 2006; Juengel et al., 2006), was infused directly into the ovary of sheep with ovarian autotransplants (Campbell et al., 2009). Treated $(n=6)$ and vehicle treated controls $(n=6)$ were infused during the early follicular phase, between 12-24 h after luteal regression and ovarian response determined by collection of samples of ovarian venous blood and transdermal ultrasound. In the absence of any change in circulating gonadotrophins or in the antral follicle population, BMP6 infusion resulted in acute but transient increases in ovarian inhibin A, androstenedione and oestradiol secretion during the second half of the infusion period (Fig. 2). Thereafter, treated animals also had an advance in the time of the LH surge by around $10 \mathrm{~h}$ and smaller pre-ovulatory follicles and 


\section{Ewe 68: Control}
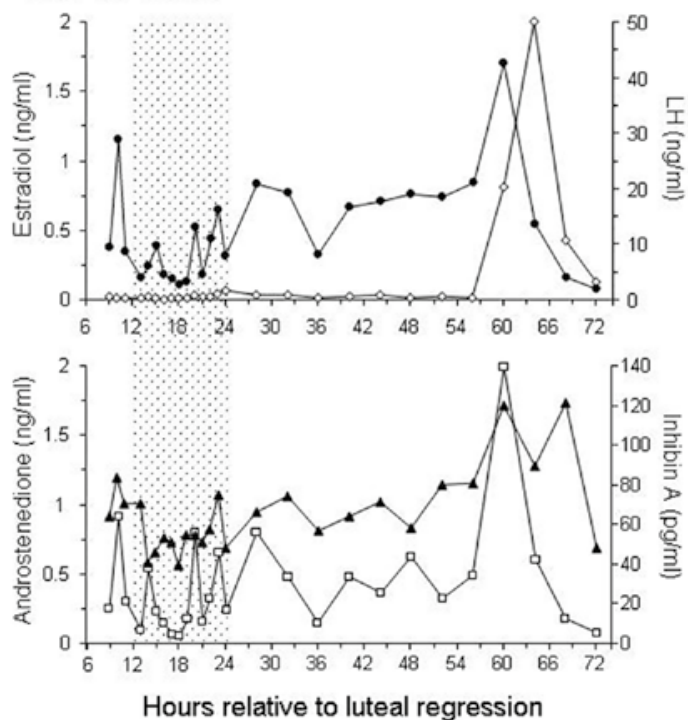

Ewe 91: Treated
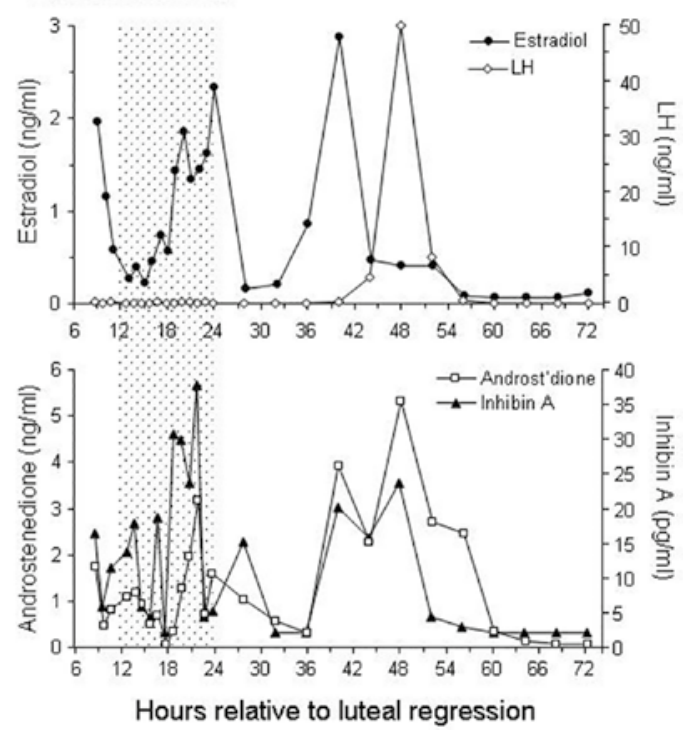

Fig. 2. Individual hormone profiles over the period of intensive blood sampling for two experimental animals, one vehicle treated control (Ewe 68) and one ewe that received BMP6 (Ewe 91) at a rate of 2 $\mu \mathrm{g} / \mathrm{h}$ for $12 \mathrm{~h}$ (hatched area) during the early follicular phase, between 12-24 h after induction of luteal regression. Upper panel for each ewe shows concentrations of LH (open diamond) and oestradiol (closed circle) whereas as lower panel shows the concentration of androstenedione (open square) and inhibin A (closed triangle) in ovarian venous plasma. Note the acute changes in ovarian hormone secretion toward the last $6 \mathrm{~h}$ of infusion in the treated animal and the subsequent advance in the time of the preovulatory surge of $\mathrm{LH}$, preceded by normal peaks of oestradiol, androstenedione and inhibin A. From Campbell et al., 2009.

corpora lutea, but without an increase in ovulation rate. This study therefore clarified that BMP6 acts as an enhancer of gonadotrophin-stimulated follicular development, with the discrepancy between in vitro and in vivo results in terms of androstenedione secretion being explained by the upregulation in ovarian inhibin secretion which is known to be a potent paracrine stimulator of thecal androgen production (Campbell and Baird, 2001; Hillier and Miro, 1993) through blockade of the inhibitory action of activin (Young and McNeilly, 2012) which is produced early in antral follicular development and also suppresses thecal androgen production (Young et al., 2012). Overall these data provided strong in vivo evidence to support the hypothesis that BMP6 is an important local regulator of ovarian function and that alterations in BMP6 cellular signalling may explain some of the effects of the FecB mutation in inducing precocious maturation of ovulatory follicles.

\section{AMH}

Although $\mathrm{AMH}$ was first identified in relation to its regulatory role in male sex differentiation, after birth this sexually dimorphic expression pattern is lost and $\mathrm{AMH}$ protein is also expressed in granulosa cells of growing follicles in the ovary (Themmen, 2005; Webb et al., 1999). In rodents (Durlinger et al., 2002; Webb et al., 1999), humans (Webb et al., 1999; Weenen et al., 2004) and 
sheep (Campbell et al., 2012) AMH protein expression starts in the columnar granulosa cells of primary follicles, is highest in granulosa cells of preantral and small antral follicles and gradually diminishes in the subsequent stages of follicle development so that $\mathrm{AMH}$ is no longer expressed during the gonadotropin-dependent terminal stages of follicle development (Fig. 1). In addition, AMH expression disappears when follicles become atretic. Amh null mice have increased rates of primordial follicle recruitment and resulting in premature ovarian failure. Further, AMH has been shown to inhibit gonadotropin-dependent growth of cultured mouse preantral follicles (Durlinger et al., 2002; Themmen, 2005; Webb et al., 1999) and in rat, pig (di Clemente et al., 1994; Webb et al., 1999) and sheep (Campbell et al., 2012) granulosa cell cultures, AMH attenuates the FSHdependent increase in aromatase activity and $\mathrm{LH}$ receptor expression. Further, in sheep we were also able to show that $\mathrm{AMH}$ is a potent inhibitor of $\mathrm{LH}$-stimulated thecal androgen synthesis in vitro (Campbell et al., 2012) and we have subsequently confirmed that theca cells do express the specific Type $2 \mathrm{AMH}$ receptor (BK Campbell, P Marsters unpublished results). Thus the available evidence supported a functional role for $\mathrm{AMH}$ in modulating both the early and terminal stages of follicle development in polyovular species. However, in relation to early follicle development the evidence was more equivocal for monovulatory species, in that the literature contained two reports which supported a role for $\mathrm{AMH}$ in the suppression of primordial follicle initiation in vitro in the human (Carlsson et al., 2006) and bovine (Gigli et al., 2005), whereas another study reported the opposite in humans (Schmidt et al., 2005).

In contrast to BMP6, direct ovarian infusion of $\mathrm{AMH}$ in ewes with ovarian autotransplants had no effect on ovarian steroid secretion or the pattern of ovulatory ovarian follicle development (Campbell et al., 2012). The converse experiment however, a knock-down utilising active immunisation in lieu of a naturally occurring mutation, had no effect on primordial follicle number but did lead to a decline in the population of gonadotrophin-responsive preantral and small antral follicles and increases in both the number of gonadotrophin-dependent antral follicles and ovulation rate (Fig. 3; (Campbell et al., 2012). In contrast to the rodent, the results indicate that AMH does not affect the rate of primordial follicle recruitment but appears to regulate the rate at which follicles progress through the gonadotropin-responsive phase, during which time it is maximally expressed. Further, the existence of a functional link between $\mathrm{AMH}$ and theca cell function in vivo was confirmed by this experiment as antral fluid levels of androstenedione were greatly elevated in AMH-immunised animals (Campbell et al., 2012) and we have subsequently shown that supplementation of sheep with the weak adrenal androgen DHEA, results in perturbation in $\mathrm{AMH}$ protein expression and an increase in the number of gonadotrophin-responsive small antral follicles. The elucidation of a functional relationship between $\mathrm{AMH}$ and $\mathrm{LH}$-stimulated thecal androgen production is significant in terms of the aetiology of common forms of anovulatory infertility in women (Franks et al., 2008) and the ability to manipulate the number of recruitable antral follicles may have therapeutic value in women who respond poorly to ovarian stimulation. Overall, this experiment also emphasises the continued value of active immunisation as a means to perturb specific local regulatory systems in domestic ruminants in the absence of natural or induced knock-down models and this conclusion is supported by the findings of Juengel et al. who showed similar ovarian responses to low and high levels of immunisation against BMP15 as observed in ewes that carried one or two copies of the FecX BMP15 mutation (Galloway et al., 2000; Juengel et al., 2004b).

Although the studies described above indicate that $\mathrm{AMH}$ represents an important local attenuator of gonadotrophin-induced somatic cell differentiation they also demonstrated that contrary to expectations, $\mathrm{AMH}$ cannot be directly implicated in the mechanism of action of the FecB mutation as in a variety of ovarian somatic cell types AMH did not signal via the BMP pathway to phosphorylate SMAD1, SMAD5 and SMAD8 (L Nicol, AS McNeilly, BK Campbell unpublished 

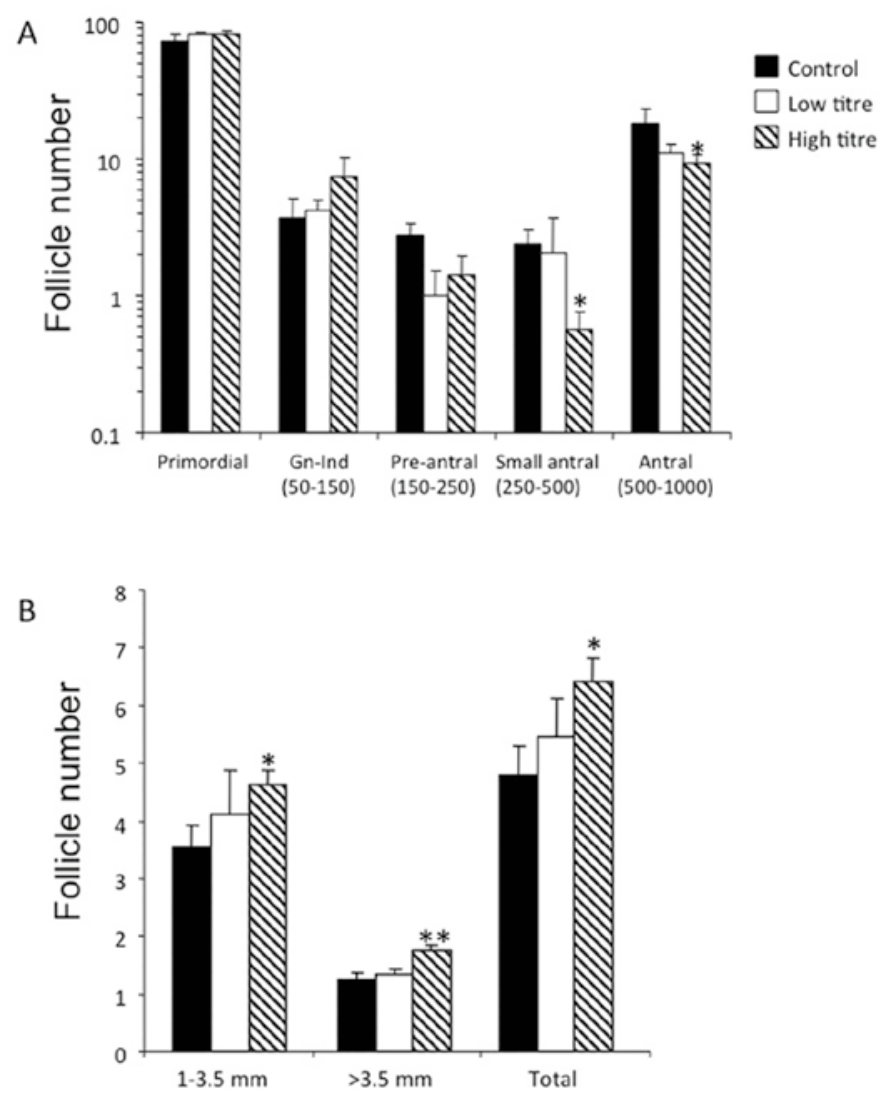

Fig. 3. Effect of active immunisation against $\mathrm{AMH}$ on the follicle population derived from either histological evaluation of the early follicle population $\leq 1 \mathrm{~mm}$ diameter in one ovary (a) or dissection of visible antral follicles $>1 \mathrm{~mm}$ diameter from the contra-lateral ovary in each animal (b). Note that immunisation in high titre animals resulted in an increase in the population of both small and large antral follicles which was associated with a decline in the population of small antral follicles less then $1 \mathrm{~mm}$ in diameter. It was concluded that $\mathrm{AMH}$, which is maximally expressed in late preantral and small antral follicles regulates the rate at which follicle pass through this gonadotrophin-responsive phase. Ovulation rates in $\mathrm{AMH}$ immunised animals were also increased. Mean $\pm \mathrm{SEM}$. ${ }^{*} \mathrm{P}<0.05,{ }^{*} \mathrm{P}<0.01$ for comparison with controls. Data published in Campbell et al., 2012.

results). Parallel studies utilising a reporter assay for multiple transcriptional responsive elements (Cignal 45-Pathway Reporter Array, SABiosciences, Crawley, U.K.) in human granulosa cell lines confirmed this result and indicated that $\mathrm{AMH}$ increases activity of a range of transcription factors including ERE, ARE, SP1 and NFAT (J Hernandez-Medrano, BK Campbell unpublished results) and further work is required to extend these studies. A further area of uncertainty in relation to $\mathrm{AMH}$ is the identity of factor(s) that stimulate its expression in granulosa cells during the very early stages of follicle development. A clue to this problem was however observed in our initial immunohistochemical (IHC) studies examining the pattern of $\mathrm{AMH}$ protein expression at different stages of follicle development (Campbell et al., 2012) where a marked inverse relationship was observed between $\mathrm{AMH}$ and aromatase protein expression. This inverse relationship was most notable in large oestrogenic follicles where the cumulus area surrounding the oocyte 
had high levels of $\mathrm{AMH}$ expression and low aromatase whereas the membrana granulosa cell layer displayed the inverse pattern of expression (Fig. 4). It appears extremely likely from this observation that maintenance of $\mathrm{AMH}$ expression in the undifferentiated cumulus cell layer of these oestrogenic follicles is due to oocyte secreted factors and follow up experiments utilising knock-down approaches in vitro have indicated functional links between granulosa cell AMH expression and BMP15, GDF9 and SOX8 (HM Picton, BK Campbell unpublished results). On the basis of these observations we have proposed the mechanistic model presented in Fig. 4 which proposes that $\mathrm{AMH}$ is upregulated in somatic cells by oocyte secreted factors (OSF) and that the down-regulation in $\mathrm{AMH}$ expression that occurs as follicles move into the gonadotrophindependent phase occurs in response to either a decrease in the concentration of OSF across this morphogen gradient and/or induction of $\mathrm{LH}$-stimulated thecal androgen secretion which acts as a negative modulator of $\mathrm{AMH}$ expression.
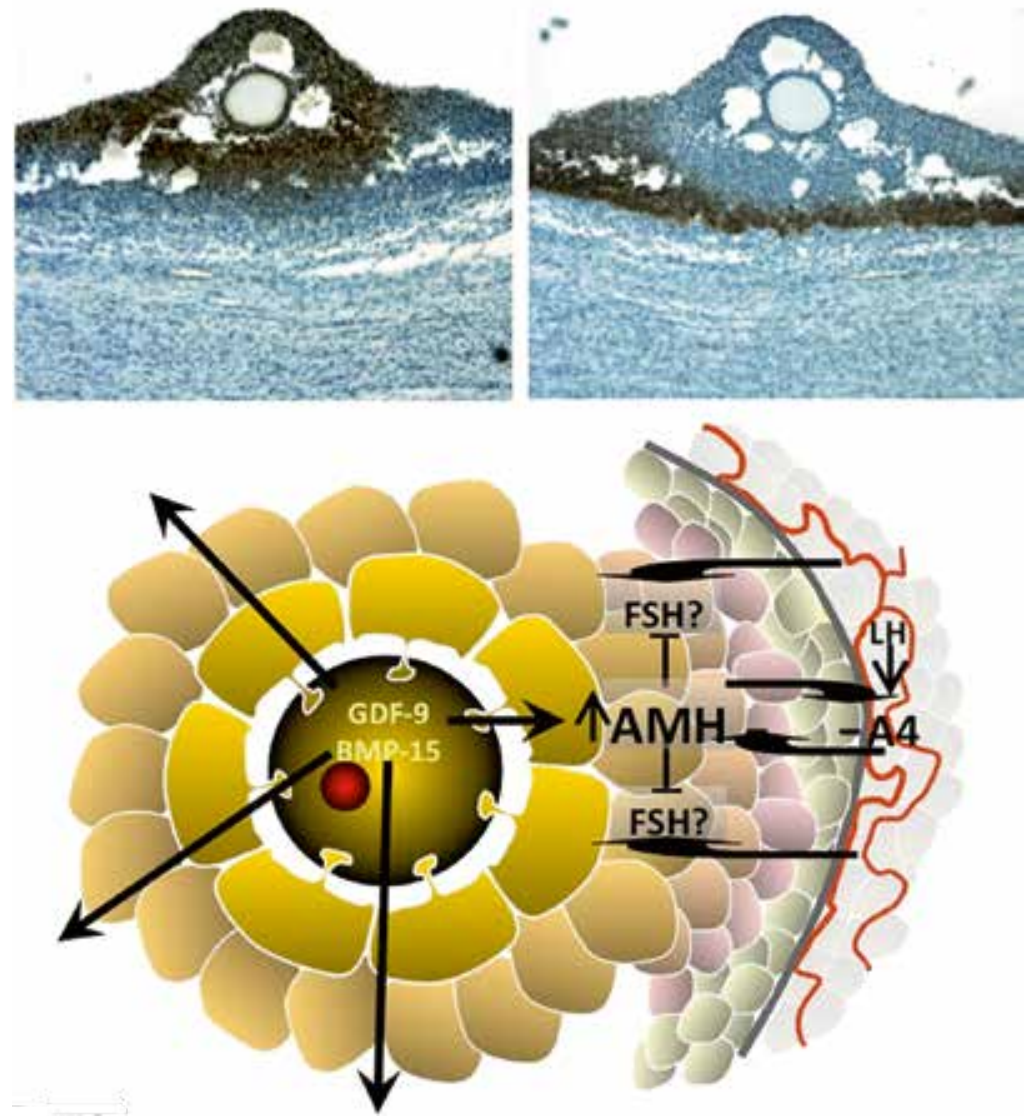

Fig. 4. Immunohistochemistry showing AMH (A) and aromatase (CYP19A1) (B) proteins in consecutive sections from the same oestrogenic large antral follicle showing that $\mathrm{AMH}$ is absent from the granulosa cell layer but present in the coronal and cumulus cells surrounding the oocyte. Note that aromatase abundance is inverse to $\mathrm{AMH}$ abundance. $\mathrm{C}$ proposed model explaining pattern expression of $\mathrm{AMH}$ shown in $\mathrm{A} / \mathrm{B}$ that that $\mathrm{AMH}$ is upregulated in somatic cells by OSF and that the down-regulation in $\mathrm{AMH}$ expression that occurs as follicles move into the gonadotrophin-dependent phase occurs in response to either a decrease in the concentration of OSF across this morphogen gradient and/or induction of $\mathrm{LH}$-stimulated thecal androgen secretion which acts as a negative modulator of AMH expression. Images A and C previously published in Campbell et al., 2012. 


\section{BMP15}

The consequences of naturally occurring mutations (Galloway et al., 2000) and/or active immunisation against BMP15 (Juengel et al., 2004b) are well described in the literature and it is clear that BMP15 is major local regulator of ovarian function. There is some evidence to support a role for BMP15 in the mechanism of action of the FecB mutation in that BMP15 expression in oocytes has been shown to be lower in FecB animals (Crawford et al., 2011) and ewes who are heterozygous for the FecX BMP15 mutation show some evidence of the precocious ovulatory follicles phenotype that is so characteristic of the Booroola (McNatty et al., 2009). In our own studies with Booroola ewes we were unable to demonstrate any effect of the FecB mutation in Alk6 on the ability of BMP15 to phosphorylate SMAD1, SMAD5 and SMAD8 (L Nicol, AS McNeilly, BK Campbell unpublished results) and parallel cell line reporter studies have also produced equivocal results (PM Marsters and BK Campbell unpublished results). The biology of BMP15, however, is complex and this is well illustrated by cell culture studies which have shown that low doses of rhBMP15 alone are mildly stimulatory to FSH-stimulated oestradiol production by granulosa cells while higher doses are markedly inhibitory. BMP15 is, however, known to form heterodimers with GDF9 (Mottershead et al., 2013) and exposure of granulosa cells to both rmGDF9 and rhBMP15 induced a 10-fold increase in oestradiol production when compared to the same doses in isolation (A Marsh, J Hernandez-Medrano, BK Campbell unpublished results).

In order to test the in vivo action of ovarian BMP15 exposure on ovulatory follicle development we have developed an alternative in situ infusion model which avoids the surgical complexity

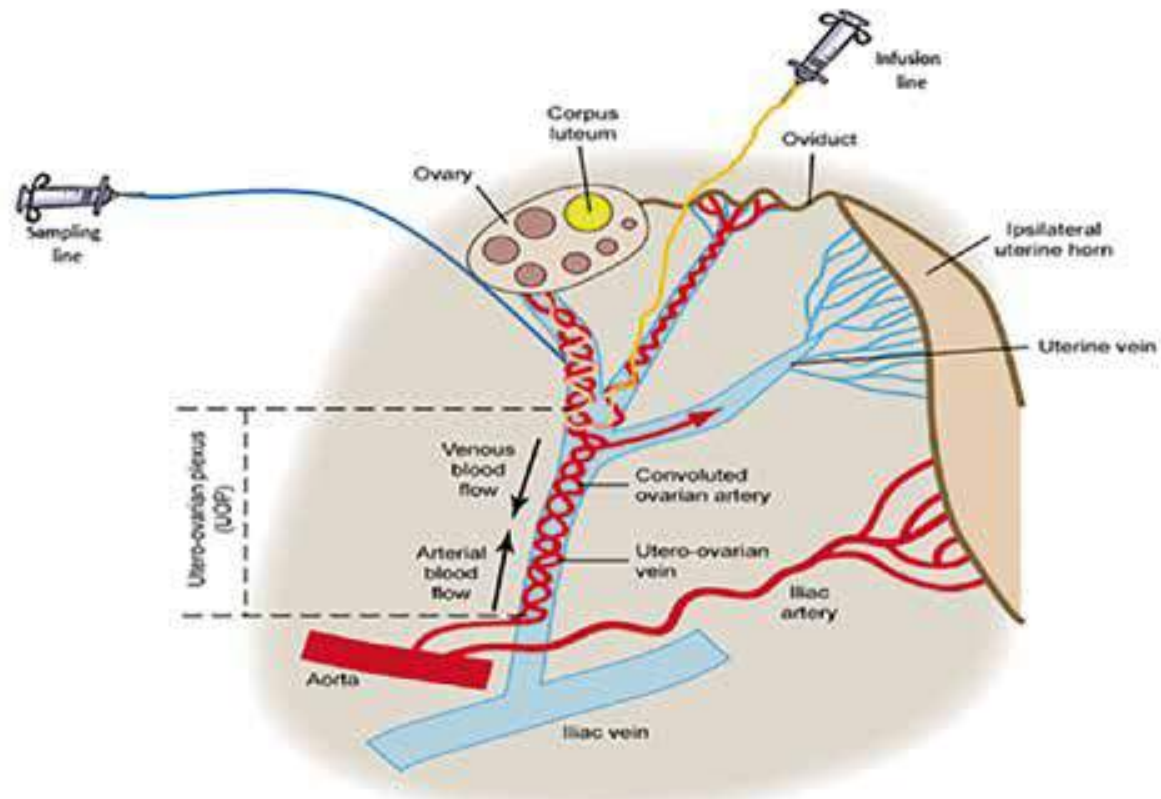

Fig. 5. Schematic diagram showing an in situ infusion model which involves the cannulation of the side-branch of the ovarian artery which supplies the broad ligament and the placement of another venous cannula in the uterine vein which is advanced so its tip lies on the common utero-ovarian vein. Cannulation of both ovaries in each animal in this manner allows one ovary to act as a vehicle only control for direct ovarian infusion of test factors via the ovarian artery following exteriorisation of all cannulae through the abdominal wall. This model avoids the surgical complexity of the ovarian autotransplant model and also allows collection of ovarian tissue for histological and molecular analysis of effects of infusion. Diagram redrawn from Lee et al.(Lee et al., 2010). 
of the ovarian autotransplant model and allows within animal comparisons. This model involves the cannulation of the side-branch of the ovarian artery which supplies the broad ligament and the placement of another venous cannula in the uterine vein which is advanced so its tip lies on the common utero-ovarian vein (Fig 5). Cannulation of both ovaries in each animal in this manner allows one ovary to act as a vehicle only control for direct ovarian infusion of test factors via the ovarian artery following exteriorisation of all cannulae through the abdominal wall. Utilising either this in situ cannulation model or the ovarian autotransplant, contrary to expectations direct ovarian infusion of rhBMP15 resulted in small but statistically significant increases in ovarian androstenedione and oestradiol secretion. This interesting result was confirmed by direct ovarian infusion of antisera raised against specific ovine BMP15 sequences (J Hernandez-Medrano, BK Campbell unpublished results). The biology of BMP15 in ruminant species therefore appears more complex than indicated by the FecX mutants and further work is required, preferably with ovine full size forms of the molecule, to elucidate its actions in vivo in relation to interactions with GDF9 and possible indirect activation of somatic cell factors such as $\mathrm{AMH}$ (Fig. 4).

In summary, the integration of in vitro and in vivo approaches has allowed us to propose a functional relationship between members of the transforming growth factor beta super family derived from both somatic cells (AMH) and the oocyte (BMP6, BMP15, GDF9) in regulating the progression of follicles through the gonadotrophin-responsive to dependent phases of follicle development. However, much more work is required to elucidate the complex biology of members of this system e.g. BMP15 and to integrate the actions of the BMP system with other major local regulatory systems such as the IGF system (Webb and Campbell, 2007b).

\section{Ruminant models of fertility restoration in women at risk of premature ovarian failure}

The domestic ruminant has been utilised for many years as an appropriate animal model for the human, due to similarities in size (sheep) and physiology, particularly in relation to the dynamics and timing of follicle development and their monovulatory nature (Campbell et al., 2003b). In the following section we will further illustrate the value of integration of both in vitro cell culture and analytical techniques with whole animal studies to ensure significant progress in the development of interventions to address human infertility. Specifically, we have used sheep as models for fertility preservation in women at risk of premature ovarian failure (POF), which occurs in 1-2\% of women (Coulam et al., 1986). For a number of reasons such as genetic predisposition (Santoro, 2003) and gonadotoxic treatment (Anderson and Wallace, 2011), the rate of follicle loss is accelerated in some women, leading to POF and early menopause ( $<40$ years old). In addition to primary infertility and amenorrhea, POF can have far-reaching clinical and psychological effects such as failure to develop secondary sexual characteristics, loss of self-confidence, depression, increased risk of osteoporosis, and heart disease (Traub and Santoro, 2010). Due to the continued improvement in childhood cancer patient survival rates, the importance of POF and its impact on human health is expanding (Anderson and Wallace, 2011). In instances when ovarian failure can be predicted, patches of ovarian cortex and the oocytes within it, can be removed and protected from damage or further deterioration in the frozen state and then autografted without vascular reanastomosis to resume development when the restoration of ovarian function is required (Donnez et al., 2013; Gosden and Nagano, 2002). The first demonstration of restoration of fertility following autografting was made in lambs in 1994 (Gosden et al., 1994) and this pioneering paper was followed by a larger scale study examining the endocrine consequences and period of restoration of fertility following cortical autografting (Baird et al., 1999). Some 5 years later the first report of a human birth from a 
woman who had received frozen cortical autografts was published (Donnez et al., 2004) and at the present time autografting of ovarian cortical tissue offers the only current proven means of restoring both fertility and ovarian function following POF in women. Although shown to be effective clinically (Donnez et al., 2013) autografting returns only a small proportion of the ovarian reserve and many of these follicles are lost due to post-graft ischaemia. This intervention is therefore less effective in older women, is associated with endocrine disturbance, involves delays of several months before ovarian function is restored and when restored the duration of ovarian function is relatively short term.

In order to try and overcome these limitations of autografting, for a number of years we have been looking at the possibility of whole ovarian cryopreservation and autotransplantation (WOCP\&TP) as a superior alternative treatment path. WOCP\&TP offers a complete solution for the long-term preservation of ovarian function and fertility in girls and women of all ages at risk of POF. This is because WOCP\&TP involves acute preservation of a whole ovary and theoretically should not diminish the ovarian reserve, result in endocrine imbalances or require a significant delay before cyclicity is restored. Further, there should be no age constraints for the long-term restoration of ovarian function.

The primary challenge with organ cryopreservation is the crucial need to preserve an intact vascular system to allow for subsequent organ revascularization and function (Pegg, 2010). All methods of cryopreservation require the replacement of the majority of the tissue water content with a cryoprotective agent (CPA). For organ preservation this has usually been achieved by vascular perfusion. While this approach creates very short diffusion distances for the CPA and water it has the disadvantage of causing microvascular damage (Pegg, 2010). From our understanding of the mechanisms of cellular damage and the mode of action of CPAs it is clear that while intra-cell injury caused by ice formation can usually be avoided, the formation of ice in extracellular locations, particularly in blood vessels, can produce serious injury and prevent organ revascularisation (Pegg, 2010). A number of different cryopreservation approaches for WOCP have been evaluated in sheep by different workers with varying degrees of success, with a large proportion of autotransplants failing after a relatively short period of time mainly due to vascular issues, although a single live birth was achieved from lambs, albeit nearly a year and half after autotransplantation (Imhof et al., 2006).

Research conducted in our laboratories has shown that cryopreservation and thawing of whole ovine ovaries using a slightly modified version of the protocol developed for cortical autografts does not significantly affect follicle viability assessed by in vitro measures (Onions et al., 2008) although some evidence of vascular damage within the ovarian pedicle was observed. Despite this, we went onto show that following slow-freezing, rapid-thawing and heterotopic autotransplantation, the reanastomosed ovarian artery of 7 out of 8 whole ovine ovaries was fully patent 7 months after autotransplantation (Onions et al., 2009). Despite patent ovarian vascular perfusion, however, follicle losses were prohibitively high and evidence of restored ovarian function was only seen in 2 out of 8 ewes. We therefore hypothesised that this follicle loss was either due to cryodamage that acute post-thaw in vitro measures of follicle viability were unable to detect, or that it was due to transient ischaemia in the ovary due to cryodamage to the ovarian vasculature. We therefore set out to both optimise the penetration of CPA through the ovary to maximise follicle survival post thaw and to maintain the integrity of the ovarian vasculature following autotransplantation. Utilising array technology we went on to show that ovarian perfusion, CPA exposure and freeze-thaw all induce apoptotic pathways and alter expression of genes associated with endothelial cell function and this finding was consistent with the observation of blood clots within the ovarian artery 7 days after WOCP\&TP (Onions et al., 2013). We therefore hypothesised that deleterious effects to the ovarian follicle 



Fig. 6. a. Image of 2 of the 4 Greyface ewes that became pregnant following WOCP\&TP with their lambs ( 1 singleton and 1 set of triplets); b. Normal appearance of ovary $(\mathrm{O})$ and vascular pedicle (VP) in a ewe 10 months after WOCP\&TP. Right hand insert is conventional ultrasound image showing presence of numerous large antral follicles in the ovary. Left hand inset shows Doppler ultrasound illustrating high levels vascular perfusion; c. Illustration of the effect of different CPA perfusion time on subsequent ovarian function. The left ovary (upper circle) was perfused with CPA for 60 minutes and right ovary (lower circle) for 10 minutes prior to WOCP\&TP in same animal. The left ovary appears normal, has well vascularised pedicle (VP) and as shown by left inset has numerous large follicles. The right ovary, despite good post-operative perfusion, was vestigial with few follicles and low blood flow (right inset) 8 months after WOCP\&TP; d. Histological image showing a cluster of numerous primordial follicles within ovary recovered 8 months after WOCP\&TP. It is estimated that $70 \%$ of primordial follicles survived this procedure and as this represents tens of thousands of follicles, this follicular cohort would enable restoration of ovarian function for several decades. Scale bar $39 \mu \mathrm{m}$. From Campbell et al., 2014.

population and surrounding ovarian tissue associated with WOCP\&TP could be attributed, at least in part, to post-operative ischaemia (within 7 days) resulting from the induction of clotting cascades within the ovarian vasculature caused by either cryo- or cytotoxic damage to the arterial endothelial cells (Onions et al., 2013). Further in vitro studies demonstrated that the period of CPA infusion was linearly related to the infusion time and that shorter periods of infusion were associated with lower levels of lactate dehydrogenase (LDH) release in vitro, used as an index of cellular damage. We therefore designed an experiment which examined the effect of cryoprotectant (CPA) perfusion time (10 vs $60 \mathrm{~min}$ ) and pre- and post-operative treatment with the anti-coagulants aspirin in combination with enoxaparine (Clexane ${ }^{\odot}$ ) or eptifibatide (Integrilin ${ }^{\odot}$ ) on ovarian function and fertility 11-23 months after WOCP\&TP. The results of this study were far beyond our expectations as combination of aspirin with additional post-operative anticoagulants resulted in acute restoration of ovarian function in 14/14 ewes within 3 weeks 
of WOCP\&TP of which 9/14 became pregnant and 4/14 gave birth to a total of 7 normal lambs (Campbell et al., 2014). There was no difference between anti-coagulants in terms of restoration of reproductive function and fertility. In contrast, the duration of CPA perfusion was highly significant with a 60 min perfusion resulting in an ovary of normal appearance and function with high rates of primordial follicle survival $(70 \%)$ and an abundant blood supply whereas ovaries perfused for $10 \mathrm{~min}$ had either resorbed completely and were vestigial (7/14) or were markedly smaller $(\mathrm{P}<0.01)$. It was concluded that both the degree of CPA penetration and maintenance of post-operative vascular patency are critical determinants of the success of WOCP\&TP and it is hoped that this work represents a major advance toward the possible development and clinical application of WOCP\&TP as a means to preserve and restore the fertility of girls and women at risk of premature ovarian failure. However, before WOCP\&TP can be used clinically, further animal model studies are required to; (i) develop optimised cryopreservation protocols for a range of different sized ovaries similar to those observed in prepubertal girls, adolescents and mature women; (ii) generate more information on the optimal type, dose and duration of post-operative anti-thrombotic therapy and (iii) examine the genetic and epigenetic normality of live offspring derived from WOCP\&TP.

\section{Concluding comments}

In the preceding review we have sought to relate some recent work in our laboratories in order to illustrate the value of integration of both in vitro cell culture and analytical techniques with whole animal studies to ensure significant progress in our understanding of the mechanisms regulating follicular development and the use of ruminants as translational models for the human. One of the major strengths of Prof Eric Lamming was his ability to understand and interact with industry bodies in order to conduct research which was targeted at real production problems and if whole animal ruminant research is to continue to thrive we need to focus on the translational nature of this work to both animal production industries and clinical medicine. Over the last 10-15 years the cost of ruminant research in the UK has increased 20-fold and similar trends seem evident in other European and North American countries. It is therefore becoming increasingly difficult to obtain funding for these types of studies and while a small proportion of studies will continue to be funded the time is perhaps right to look to the types of international collaborations encouraged by bodies such as this Ruminant Reproduction meeting that Eric Lamming helped to establish, in order to maintain the balance and integration of high quality laboratory research with whole animal studies. Despite the costs of these whole animal approaches, the need for increasing ruminant production while minimising environmental impact has never been greater because of global food security and climate change challenges. Therefore the integration of human and animal research has a double benefit for the health and wealth implications of both developed and developing countries.

\section{Acknowledgments}

The work presented in this paper was funded by the MRC, BBSRC and the University of Nottingham. The authors would like to thank the staff at the Marshall Building, University of Edinburgh; Mrs. Joan Docherty, Miss Marjorie Thompson and Mr. John Hogg for their care of the animals and Mrs Catherine Pincott-Allen, Ms Linda Nicol, Mrs Li Guo, Mrs Anne Skinner, Marcus Mitchell, Mick Baker and Ms Nicky Farrar technical assistance and the input of Adam Marsh, Feda Aljaser and Shreena Taylor into some of the studies referred to in this review. 


\section{Bibliography}

Anderson RA and WH Wallace 2011 Fertility preservation in girls and young women. Clinical Endocrinology (Oxford) 75 409-419.

Baird DT 1983 Factors regulating the growth of the preovulatory follicle in the sheep and human. Journal Reproduction and Fertility 69 343-352.

Baird DT, R Webb, BK Campbell, LM Harkness and RG Gosden 1999 Long-term ovarian function in sheep after ovariectomy and transplantation of autografts stored at -196 C. Endocrinology 140 462-471.

Cahill L 1981 Folliculogenesis in the sheep as influenced by breed, season and oestrous cycle. Journal Reproduction and Fertility Supplement 30 135-142.

Campbell B, R Scaramuzzi and R Webb 1995 Control of antral follicle development and selection in sheep and cattle. Journal Reproduction and Fertility Supplement 49 335-350.

Campbell B, R Scaramuzzi and R Webb 1996 Induction and maintenance of oestradiol and immuno-reactive inhibin production with FSH by ovine granulosa cells cultured in serum free media. Journal Reproduction and Fertility 106 7-16.

Campbell BK and DT Baird 2001 Inhibin A is a follicle stimulating hormone-responsive marker of granulosa cell differentiation, which has both autocrine and paracrine actions in sheep. Journal of Endocrinology 169 333-345.

Campbell BK, DT Baird, CJ Souza and R Webb 2003a The FecB (Booroola) gene acts at the ovary: in vivo evidence. Reproduction 126 101-111.

Campbell BK, DT Baird and R Webb 1998 Effects of dose of $\mathrm{LH}$ on androgen production and luteinization of ovine theca cells cultured in a serum-free system. Journal Reproduction and Fertility 112 69-77.

Campbell BK, M Clinton and R Webb 2012 The role of antiMullerian hormone (AMH) during follicle development in a monovulatory species (sheep). Endocrinology 153 4533-4543.

Campbell BK et al 2014 Restoration of ovarian function and natural fertility following the cryopreservation and transplantation of whole adult ovaries. Human Reproduction (in press).

Campbell BK, NR Kendall and DT Baird 2009 Effect of direct ovarian infusion of bone morphogenetic protein 6 (BMP6) on ovarian function in sheep. Biology of Reproduction 81 1016-1023.

Campbell BK et al 2003b Domestic ruminants as models for the elucidation of the mechanisms controlling ovarian follicle development in humans. Reproduction Supplement 61 429-443.

Campbell BK, CJ Souza, AJ Skinner, R Webb and DT Baird 2006 Enhanced response of granulosa and theca cells from sheep carriers of the FecB mutation in vitro to gonadotropins and bone morphogenic protein-2, -4 and -6. Endocrinology 147 1608-1620.

Carlsson IB et al 2006 Anti-Mullerian hormone inhibits initiation of growth of human primordial ovarian follicles in vitro. Human Reproduction 21 2223-2227.

Coulam CB, SC Adamson and JF Annegers 1986 Incidence of premature ovarian failure. Obstetrics and Gynecology
67 604-606.

Crawford JL et al 2011 Oocytes in sheep homozygous for a mutation in bone morphogenetic protein receptor $1 \mathrm{~B}$ express lower mRNA levels of bone morphogenetic protein 15 but not growth differentiation factor 9 . Reproduction 142 53-61.

di Clemente $\mathbf{N}$ et al 1994 Inhibitory effect of $\mathrm{AMH}$ upon the expression of aromatase activity and $\mathrm{LH}$ receptors by cultured granulosa cells of rat and porcine immature ovaries. Endocrine 2 553-558.

Donnez J et al 2004 Livebirth after orthotopic transplantation of cryopreserved ovarian tissue. Lancet 364 1405-1410.

Donnez J et al 2013 Restoration of ovarian activity and pregnancy after transplantation of cryopreserved ovarian tissue: a review of 60 cases of reimplantation. Fertility and Sterility 99 1503-1513.

Dufour J, L Cahill and P Mauleon 1979 Short and long-term effects of hypophysectomy and unilateral ovariectomy on ovarian follicular populations in sheep. Journal Reproduction and Fertility 57 301-309.

Durlinger AL, JA Visser and AP Themmen 2002 Regulation of ovarian function: the role of anti-Mullerian hormone. Reproduction 124 601-609.

Franks S, J Stark and K Hardy 2008 Follicle dynamics and anovulation in polycystic ovary syndrome. Human Reproduction Update 14 367-378.

Galloway SM et al 2000 Mutations in an oocyte-derived growth factor gene (BMP15) cause increased ovulation rate and infertility in a dosage-sensitive manner. Nature Genetics 25 279-283.

Gigli I, RA Cushman, CM Wahl and JE Fortune 2005 Evidence for a role for anti-Mullerian hormone in the suppression of follicle activation in mouse ovaries and bovine ovarian cortex grafted beneath the chick chorioallantoic membrane. Molecular Reproduction and Development 71 480-488.

Glister C, SL Richards and PG Knight 2005 Bone morphogenetic proteins (BMP) $-4,-6$ and -7 potently suppress basal and luteinizing hormone-induced androgen production by bovine theca interna cells in primary culture: could ovarian hyperandrogenic dysfunction be caused by a defect in thecal BMP signaling? Endocrinology 146 1883-1892.

Gosden R and M Nagano 2002 Preservation of fertility in nature and ART. Reproduction 123 3-11.

Gosden RG, DT Baird, JC Wade and R Webb 1994. Restoration of fertility to oophorectomized sheep by ovarian autografts stored at -196 degrees C. Human Reproduction 9 597-603.

Hillier SG and F Miro 1993 Inhibin, activin and follistatin. Potential roles in ovarian physiology. Annals New York Academy of Science 687 29-38.

Imhof $\mathbf{M}$ et al 2006 Orthotopic microvascular reanastomosis of whole cryopreserved ovine ovaries resulting in pregnancy and live birth. Fertility and Sterility 85 Supplement 1 1208-1215.

Juengel JL et al 2004a Physiology of GDF9 and BMP15 signalling molecules. Animal Reproduction Science 82-83 447-460. 
Juengel JL, NL Hudson, L Whiting and KP McNatty 2004b Effects of immunization against bone morphogenetic protein 15 and growth differentiation factor 9 on ovulation rate, fertilization and pregnancy in ewes. Biology of Reproduction 70 557-561.

Juengel JL et al 2006 The role of bone morphogenetic proteins 2, 4, 6 and 7 during ovarian follicular development in sheep: contrast to rat. Reproduction 131 501-513.

Knight PG and C Glister 2006 TGF-beta superfamily members and ovarian follicle development. Reproduction 132 191-206.

Lee J et al 2010. Transport of prostaglandin F(2alpha) pulses from the uterus to the ovary at the time of luteolysis in ruminants is regulated by prostaglandin transportermediated mechanisms. Endocrinology 151 3326-3335.

McNatty KP et al 2009 Gonadotrophin-responsiveness of granulosa cells from bone morphogenetic protein 15 heterozygous mutant sheep. Reproduction 138 545-551.

McNatty KP and KM Henderson 1987 Gonadotrophins, fecundity genes and ovarian follicular function. Journal of Steroid Biochemistry 27 365-373.

McNatty KP et al 2005. Oocyte-expressed genes affecting ovulation rate. Molecular and Cellular Endocrinology 234 57-66.

Miyazono K, S Maeda and T Imamura 2005 BMP receptor signaling: transcriptional targets, regulation of signals and signaling cross-talk. Cytokine and Growth Factor Reviews 16 251-263.

Mottershead DG et al 2013. Growth differentiation factor 9:bone morphogenetic protein 15 (GDF9:BMP15) synergism and protein heterodimerization. Proceedings National Academy of Sciences 110 E2257.

Mulsant $\mathbf{P}$ et al 2001. Mutation in bone morphogenetic protein receptor-IB is associated with increased ovulation rate in Booroola Merino ewes. Proceedings National Academy of Sciences 98 5104-5109.

Onions VJ, MRP Mitchell, BK Campbell and R Webb 2008 Ovarian tissue viability following whole ovine ovary cryopreservation: assessing the effects of sphingosine-1phosphate inclusion. Human Reproduction 23 606-618.

Onions VJ, R Webb, AS McNeilly and BK Campbell 2009 Ovarian endocrine profile and long-term vascular patency following heterotopic autotransplantation of cryopreserved whole ovine ovaries. Human Reproduction 24 2845-2855.

Onions VJ, R Webb, C Pincott-Allen, HM Picton and BK Campbell 2013 The effects of whole ovarian perfusion and cryopreservation on endothelial cell-related gene expression in the ovarian medulla and pedicle. Molecular Human Reproduction 19 205-215.

Pegg DE 2010 The relevance of ice crystal formation for the cryopreservation of tissues and organs. Cryobiology 60 S36-44.

Picton HM, MA Danfour, SE Harris, EL Chambers and J Huntriss 2003 Growth and maturation of oocytes in vitro. Reproduction Supplement 61 445-462.

Picton HM, SE Harris, W Muruvi and EL Chambers 2008 The in vitro growth and maturation of follicles. Reproduction $136703-715$.
Santoro N 2003 Mechanisms of premature ovarian failure. Annals Endocrinology (Paris) 64 87-92.

Schmidt KL, N Kryger-Baggesen, AG Byskov and CY Andersen 2005 Anti-Mullerian hormone initiates growth of human primordial follicles in vitro. Molecular and Cellular Endocrinology 234 87-93.

Shi Y and J Massague 2003 Mechanisms of TGF-beta signaling from cell membrane to the nucleus. Cell 113 685-700.

Souza CJ et al 2001 The Booroola (FecB) phenotype is associated with a mutation in the bone morphogenetic receptor type $1 \mathrm{~B}$ (BMPR1B) gene. Journal of Endocrinology 169 R1-6.

Themmen AP 2005 Anti-Mullerian hormone: its role in follicular growth initiation and survival and as an ovarian reserve marker. Journal of the National Cancer Institute Monographs 18-21.

Tingen C, A Kim and TK Woodruff 2009 The primordial pool of follicles and nest breakdown in mammalian ovaries. Molecular Human Reproduction 15 795-803.

Traub ML and N Santoro Reproductive aging and its consequences for general health. Annals New York Academy of Science 1204 179-187.

Turnbull K, A Braden and P Mattner 1977 The pattern of follicular growth and atresia in the ovine ovary. Australian Journal of Biological Sciences 30 229-241.

Webb R and B Campbell 2007a Development of the dominant follicle: mechanisms of selection and maintenance of oocyte quality. Reproduction Supplement 64 141-164.

Webb $\mathbf{R}$ et al 1999 Molecular mechanisms regulating follicular recruitment and selection. Journal Reproduction and Fertility Supplement 53 33-48.

Webb R and BK Campbell 2007b.Development of the dominant follicle: mechanisms of selection and maintenance of oocyte quality. Journal Reproduction and Fertility Supplement 64 141-163.

Weenen C et al 2004 Anti-Mullerian hormone expression pattern in the human ovary: potential implications for initial and cyclic follicle recruitment. Molecular Human Reproduction 10 77-83.

Wilson T et al 2001. Highly prolific Booroola sheep have a mutation in the intracellular kinase domain of bone morphogenetic protein IB receptor (ALK-6) that is expressed in both oocytes and granulosa cells. Biology of Reproduction 64 1225-1235.

Young JM et al 2012 Activin B is produced early in antral follicular development and suppresses thecal androgen production. Reproduction 143 637-650.

Young JM and AS McNeilly 2010 Theca: the forgotten cell of the ovarian follicle. Reproduction 140 489-504.

Young JM and AS McNeilly 2012 Inhibin removes the inhibitory effects of activin on steroid enzyme expression and androgen production by normal ovarian thecal cells. Journal Molecular Endocrinology 48 49-60. 
